\title{
Complete genome sequence of a Tobacco necrosis virus D isolate from olive trees
}

\section{Source: ARCHIVES OF VIROLOGY Volume: 154 Issue: 7 Pages: 1169} 1172 Published: JUL 2009

Author(s): Cardoso JMS (Cardoso, Joana M. S.)2, Felix MR (Felix, Maria R.)2, Clara MIE (Clara, Maria I. E.)2, Oliveira S (Oliveira, Solange) 1,3

Received: 2 April 2009 / Accepted: 27 May 2009

_ Springer-Verlag 2009

The first complete genome sequence of an isolate of Tobacco necrosis virus D (TNV-D; genus Necrovirus, family Tombusviridae) was that of a bean isolate from England [4]. The complete nucleotide sequence of another TNV-D isolate, an Hungarian isolate from tobacco plants (TNV-Dн), revealed a similar genomic organization [11]. The single-stranded positive-sense RNA genome of TNV-D is about $3.8 \mathrm{~kb}$ in size and contains six open reading frames (ORFs).

Times Cited: 2 References: 15 Citation Map Document Type: Article

Language: English

KeyWords Plus: COMPLETE NUCLEOTIDE-SEQUENCE; OLEA-EUROPAEA L; STRAIN-D; SOFTWARE; DATABASE; RNA

Reprint Address: Oliveira, S (reprint author), Univ Evora, Dept Biol, Nucleo Mitra, Apartado 94, P7002554 Evora, Portugal

Addresses: 1. Univ Evora, Dept Biol, Nucleo Mitra, P-7002554 Evora, Portugal 2. Univ Evora, Lab Plant Virol, Inst Mediterranean Agr Sci, P-7002554 Evora, Portugal 3. Univ Evora, Soil Microbiol Lab, Inst Mediterranean Agr Sci, P-7002554 Evora, Portugal

E-mail Addresses: ismo@uevora.pt 